\title{
Nanoparticles for photoacoustic imaging
}

\author{
Xinmai Yang, ${ }^{1}$ Erich W. Stein, ${ }^{1}$ S. Ashkenazi ${ }^{2}$ and Lihong V. Wang ${ }^{1, *}$
}

\begin{abstract}
Nanoparticles have been designed and applied as contrast enhancers in various optical imaging techniques, such as optical coherence tomography, fluorescence imaging, and optical reflectance microscopy. As an emerging hybrid imaging modality, photoacoustic imaging (PAI) has also benefited from the application of these nanoparticle-based contrast agents. We review this rapidly growing field and describe the applications of nanoparticles in PAI. Particular focus is given to nanoparticles whose absorption mechanism is based on surface plasmon resonance, including gold nanoshells, nanorods, and nanocages. Dye-embedded nanoparticles are also reviewed. Specifically, the design and application of each nanoparticle-based contrast agent in relation to the field of PAI are detailed. (๑) 2009 John Wiley \& Sons, Inc. WIREs Nanomed Nanobiotechnol 2009 1360-368
\end{abstract}

$\mathrm{P}$ hotoacoustic imaging (PAI), also called optoacoustic or thermoacoustic imaging, has emerged as a promising non-invasive imaging modality ${ }^{1-6}$ that combines the spectral selectivity of molecular excitation by laser light with the high resolution of ultrasound imaging. Currently, two PAI techniques are actively studied: photoacoustic tomography (PAT) and photoacoustic microscopy (PAM). ${ }^{6-8}$ Typically, PAT utilizes either an ultrasound detector array or single scanned detector (to mimic an array) and an inverse algorithm to reconstruct cross-sectional or three-dimensional images of biological tissue. In contrast to PAT, PAM typically uses a raster-scanned focused ultrasonic detector coupled with confocal optical illumination and requires no reconstruction algorithm. Additionally, the optical-ultrasonic confocal configuration improves the signal-to-noise ratio.

PAI takes advantage of the high optical contrast of biological tissue. Because of the high optical absorption of hemoglobin over a broad optical spectrum, PAI has been proven to be a powerful tool for imaging blood vessels. PAI has also been used to track the uptake, delivery, and excretion of optical tracers in vivo. ${ }^{9-11}$. Thus, PAI is suitable for monitoring both endogenous and exogenous optical contrast agents such as hemoglobin and organic dyes, respectively. ${ }^{12}$

\footnotetext{
*Correspondence to: lhwang@biomed.wustl.edu

${ }^{1}$ Department of Biomedical Engineering, Washington University, St. Louis, MO 63130, USA

${ }^{2}$ Department of Biomedical Engineering, University of Michigan, Ann Arbor, MI 48109, USA

DOI: $10.1002 /$ wnan.042
}

Advances in nanotechnology have produced significant contributions by means of nanoparticle-based contrast agents, which vary in diameter from $1 \mathrm{~nm}$ to several hundred nanometers. ${ }^{13,14}$ Furthermore, the surface characteristics of these nanoparticles can be engineered to enhance biocompatibility.

Nanoparticles designed for high photoacoustic contrast can be classified into two major types based on the physical mechanism of light absorption: particles based on surface plasmon resonance (SPR) and dye-containing nanoparticles. For SPR applications, nanoscale metallic films, most commonly gold, are deposited onto the nanoparticle surface. Peak absorption can be tuned throughout the visible and near infrared (NIR) regions by simply controlling the physical dimensions of the metallic layer. ${ }^{15,16}$ Furthermore, gold surfaces exhibit excellent biocompatibility and are a simple means to add additional surface functionalities. Dye-containing nanoparticles, however, utilize a high payload of NIR organic dyes to enhance optical absorption. For example, indocyanine green (ICG), an Food and Drug Administration (FDA)-approved dye, has been encapsulated within nanoparticles and used as a contrast enhancer for PAI. ${ }^{17}$

The use of nanoparticle-based contrast agents greatly extends PAI applications. (1) It allows PAI to image deeper within tissue with enhanced contrast. Nanoparticles are usually designed with peak absorption in the NIR region, where optical attenuation of tissue is relatively low, affording deep light penetration. (2) Targeting peptides and antibodies can be conjugated to the nanoparticle surface for cell specific 


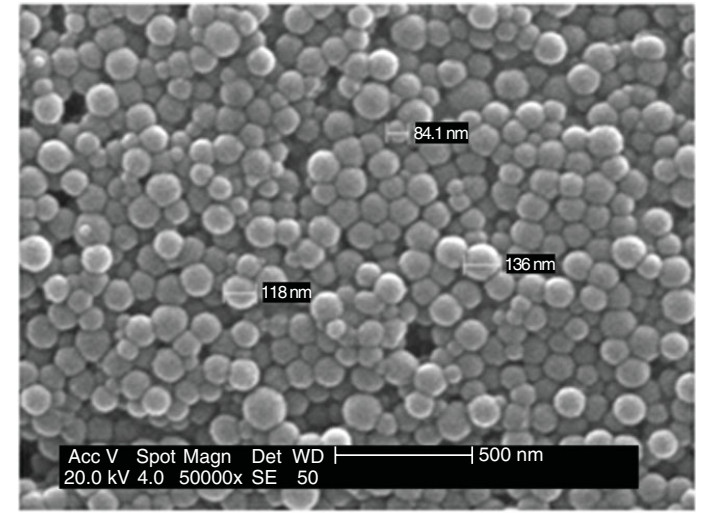

(a)

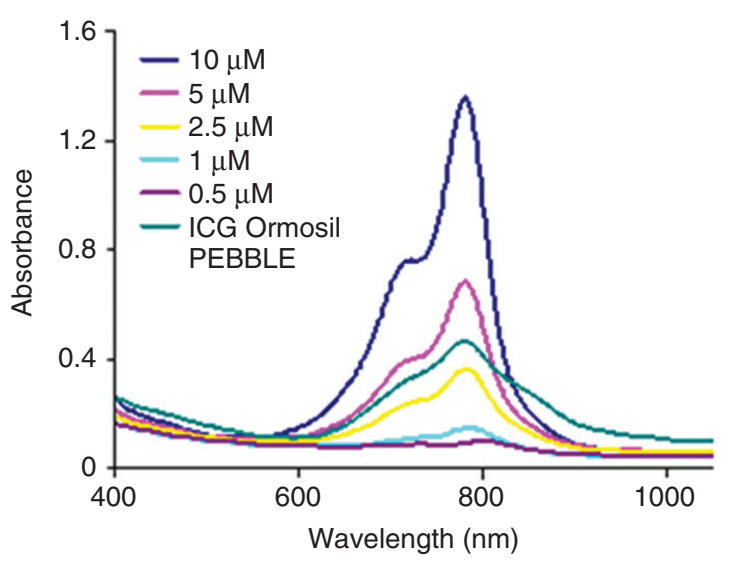

(b)

FIG URE 1 | SEM image of ICG PEBBLE (a) and optical absorption of ICG PEBBLEs and ICG free dye at different concentrations (b). Comparison yields a mean loading of 23000 ICG molecules per nanoparticles (Reproduced with permission from Ref 42. Copyright 2007 SPIE).

contrast and molecular imaging. (3) Potentially, PAI can be easily combined with photothermal therapy, because both techniques share similar mechanisms.

In the following, we will review the design, synthesis, characterizations, and application of different types of nanoparticle-based PAI contrast agents. The focus will be on gold nanoshells, gold nanorods, gold nanocages, and ICG-doped nanoparticles, which are contrast agents studied in PAI.

\section{CHARACTERIZATION OF NANOPARTICLES IN PAI}

Of the four kinds of nanoparticles used in PAI, nanoshells were the first to be adopted. Nanoshells usually consist of a dielectric core coated by a conductive, nanometer-thick metallic shell, ${ }^{18}$ usually gold.
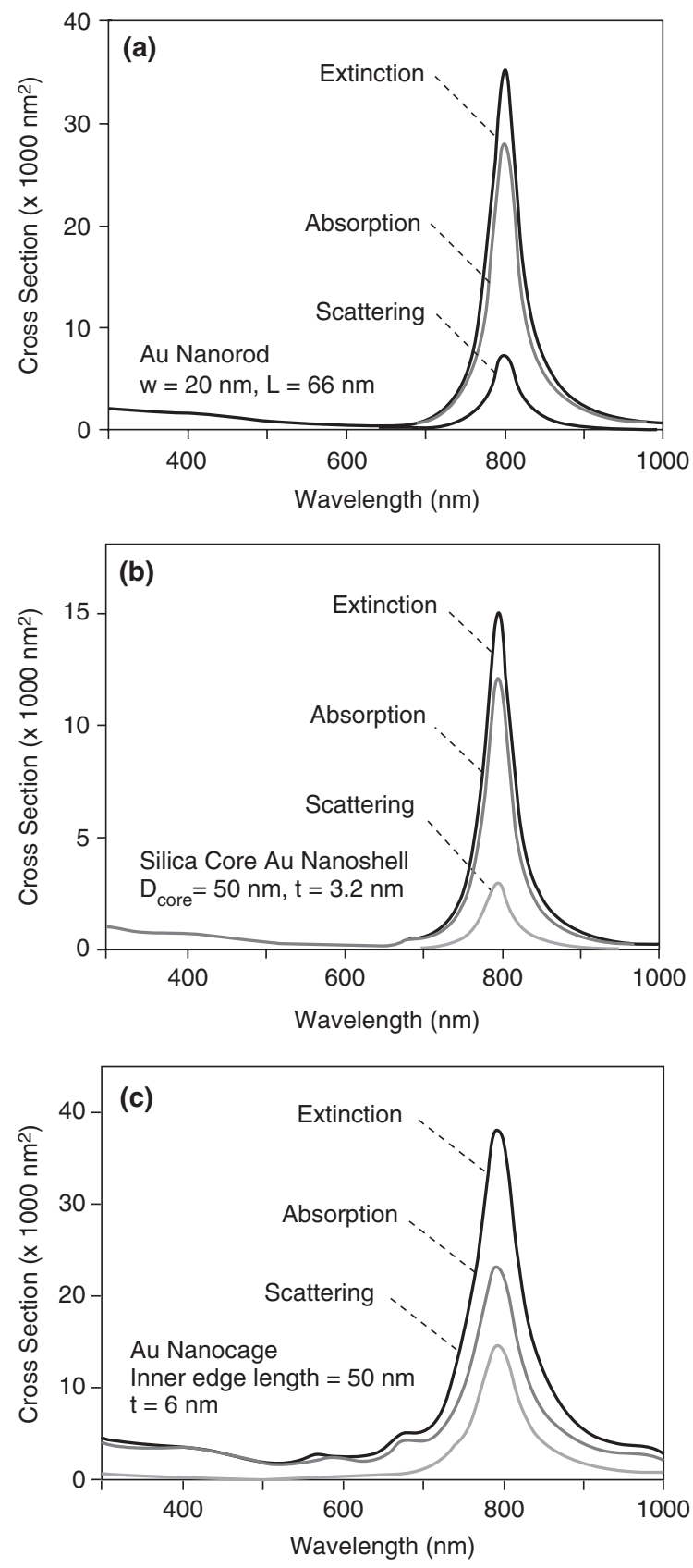

FIG URE 2 | (Comparison of the optical spectra calculated using DDA for gold nanorods, nanoshells, and nanocages. For all the structures, the SPR peak was tuned to exactly $800 \mathrm{~nm}$. (a) The gold nanorod has a width of $w=20 \mathrm{~nm}$ and length $\mathrm{L}=66 \mathrm{~nm}$. (b) The nanoshell has a silica core of $50 \mathrm{~nm}$ in diameter and shell thickness of $3.2 \mathrm{~nm}$. (c) The nanocage has a water core with an inner edge length of $50 \mathrm{~nm}$ and wall thickness of $6 \mathrm{~nm}$. (Reproduced with permission from Ref 33. Copyright 2006 The Royal Society of Chemistry).

Consequently, alterations in core size and/or shell thickness shift the SPR, allowing nanoparticle optical properties (absorption and scattering) to be tuned 
over the visible and NIR spectral regions. ${ }^{19,20}$ This level of tunability is a highly attractive property, affording biomedical applications from sensing to imaging. ${ }^{14,21}$ Particularly, nanoshells have been engineered with absorption peaks ranging from 600 to $900 \mathrm{~nm}$, a spectral region lying within the tissue 'optical window' where absorption is minimal and optical penetration is maximal. ${ }^{22}$ In additional to favorable spectral characteristics, nanoshells have been proven to be optically robust, exhibiting resistance to chemical/thermal denaturation and photobleaching, commonplace effects that compromise the longevity of NIR dyes. ${ }^{23,24}$ Furthermore, nanoshells comprising gold offer a straightforward means to chemically modify surface characteristics (and modulate host response when used in vivo) by grafting on functional groups ranging from polyethylene glycols to integrins. ${ }^{25}$

Gold nanorods represent another kind of nanoparticles whose absorption mechanism is based on SPR. Unlike nanoshells, nanorods exhibit cylindrical symmetry, and simple changes in particle symmetry can significantly alter SPR characteristics. Like nanoshells, nanorods have strong resonance optical absorption, a property attributed to their shape. ${ }^{26,27}$ In contrast, nanorods can be made smaller (tens of nanometers) than nanoshells, increasing their efficiency in targeting specific cellular receptors.

With a more complicated shape, gold nanocages represent a novel class of optically tunable nanoparticles with cuboidal symmetry. ${ }^{28-30}$ It has been demonstrated that nanocages can be melted by a camera flash discharge because of their high optical absorption. ${ }^{31}$ They are prepared by a simple galvanic replacement reaction between $\mathrm{Ag}$ nanocubes and $\mathrm{HAuCl}_{4}$. By adjusting the amount of $\mathrm{HAuCl}_{4}$ added, the SPR peaks of the resultant $\mathrm{Au}$ nanocages can be precisely tuned throughout the visible and NIR regions. For in vivo use, gold nanoparticles are typically PEGylated prior to introduction into the host.

In addition to regular geometries such as shells and rods, other SPR nanoparticles with even more complex shapes have been designed, such as multipods, star shapes, lumps, etc. ${ }^{32}$ These nanoparticles can display well-defined peaks in the ensemble spectra, which are determined by their shapes. However, to date, there is no published work on the application of these nanoparticles in PAI.

Various methods have been demonstrated for preparing gold nanoparticles and have been documented else where, and interested readers are referred to Refs ${ }^{31,33-37}$ for details.

Nanoparticles that do not rely on SPR absorption have also been proposed for PAI. Nanoparticles (100 nm in diameter) containing ICG have been developed as a contrast agent for PAI based on photonic explorers for biomedical use by biologically localized embedding (PEBBLE) technology, ${ }^{38-40}$ using organically modified silicate (ormosil) as a matrix (see Figure $1(\mathrm{a}))^{41-43}$ This technology, originally developed for sensing and therapeutic applications, allows the incorporation of sensor dyes, imaging contrast agents, drugs, photosensitizers for photodynamic therapy (PDT), and cell-targeting ligands in a nanosize biocompatible particle. ICG has strong optical absorption in the NIR region, and contrast enhancement in PAI by ICG in its free dye form has been demonstrated. ${ }^{10}$ However, free dye ICG has a half-life of only several minutes inside the blood stream. Dye encapsulation in nanoparticles significantly increases the circulation time in blood, ${ }^{42}$ allowing for an extended imaging period. Dye encapsulation has several other advantages. First, the nanoparticle surface can be engineered for specific purposes, for example, incorporating a targeting moiety for selective contrast enhancement. Second, superior contrast is achieved because of the high dye concentration in the nanoparticle. Finally, encapsulation in a nanoparticle stabilizes ICG dye molecules against the effects of an aqueous medium and other destabilizing effects from the biological environment. More details about the preparation can be found in Ref. ${ }^{42}$.

Optical absorption spectra of ICG-ormosil PEBBLEs (Figure 1(b)) indicate efficient entrapping of ICG dye in the nanoparticle matrix $(23,000$ dye molecules per single nanoparticle). The matrix's high affinity for ICG acts to stabilize the dye against aggregation and to retain its high optical absorption even at extremely high dye concentrations. The ormosil encapsulation is also efficient in protecting the dye from the environment. ICG-ormosil PEBBLEs showed improved stability in aqueous solution compared with free ICG dye.

\section{THEORETICAL COMPARISONS OF OPTICAL ABSORPTION}

Unlike most traditional optical imaging techniques, in PAI, the signal from photoacoustic effect is proportional to optical absorption. Naturally, the most critical requirement for PAI contrast agents is high optical absorption. A study by $\mathrm{Hu}$ et al. ${ }^{33}$ compared the different nanostructures in the context of biomedical applications, where the exact magnitudes of both scattering and absorption are important. Figure 2 compares the absorption, scattering, and extinction spectra calculated for gold nanorods, nanoshells, and nanocages using the discrete dipole approximation (DDA) method. In these results, the dimensions of the 
structures were adjusted to tune their SPR peaks to $800 \mathrm{~nm}$, which is the typical wavelength chosen in the NIR range in biomedical applications. This wavelength requires gold nanorods with an aspect ratio of 3.3 and a fixed width of $20 \mathrm{~nm}$, gold nanoshells on silica cores with a core diameter of $50 \mathrm{~nm}$ and shell thickness of $3.2 \mathrm{~nm}$, and gold nanocages with an inner edge length of $50 \mathrm{~nm}$ and wall thickness of $6 \mathrm{~nm}$. The comparisons are based on cross-sections. It is worth noting that gold nanocages and nanorods have much larger absorption and scattering cross-sections than gold nanoshells: i.e., $\mathrm{C}_{\text {cage }} \approx \mathrm{C}_{\text {rod }}>t \mathrm{C}_{\text {shell }}$.

A side by side comparison between ICG-ormosil PEBBLEs and SPR nanoparticles is not available in the literature. However, because ICG-ormosil PEBBLEs encapsulate $\sim 23,000$ dye molecules per single nanoparticle, the absorption of these nanoparticles may be at the same order of magnitude as SPR particles. (The absorption of gold nanoparticles is about four to five orders of magnitude stronger comparing free ICG).

\section{ON-GOING WORK}

The initial application of nanoshells as a photoacoustic contrast agent was to increase the NIR contrast of cortical blood vessels in the in vivo rat brain. ${ }^{9}$
Nanoshells comprising PEGylated surfaces (half-life of $\sim 3.7 \mathrm{~h}$ in blood) with a mean diameter of $\sim 135 \mathrm{~nm}$ and peak absorption of $800 \mathrm{~nm}$ were injected into the tail vein of Sprague-Dawley rats. Cortical vasculature was non-invasively imaged using PAT following nanoshell injections (three in total). Successive image acquisition was used to quantify circulation dynamics, including clearance, in the cortical blood stream (Figure 3). Comparison of the image acquired using only endogenous contrast (Figure 3(a)) to that acquired after the third administration of nanoshells (Figure $3(\mathrm{~b})$ ) demonstrated a substantial increase in vascular contrast because of the presence of nanoshells in the blood stream. The difference in the post- and preinjection images (Figure 3(c)) depicted the nanoshell distribution within the cortical vasculature. Furthermore, the mean photoacoustic amplitude, which is proportional to volume-specific optical absorption, of the cortical vasculature for each successive image was calculated and plotted versus time (Figure 3(d)). Following three sequential nanoshell doses, these data depicted a $63 \%$ increase in blood vessel absorption. This work illustrated the feasibility of using nanoshells in vivo as an NIR contrast agent in PAI, with particular implications in enhancing the sensitivity of cortical blood volume measurements in non-invasive studies of small animal brain function. ${ }^{5}$ While this
FIGURE 3 | (a) Noninvasive in vivo photoacoustic image of rat cortical vasculature acquired using $800 \mathrm{~nm}$ irradiation before administration of nanoshells as a NIR contrast agent. MF: median fissure. (b) Photoacoustic image acquired $\sim 20$ min post-injection of the third dose of nanoshells. (c) Difference image obtained by subtracting pre- and post-injection images (i.e. Image $C=$ Image B-Image A). (d) Mean optical absorption of cortical vasculature normalized to the mean optical absorption prior to nanoshells administration. (Reproduced with permission from Ref 9. Copyright 2004 American Chemical Society).

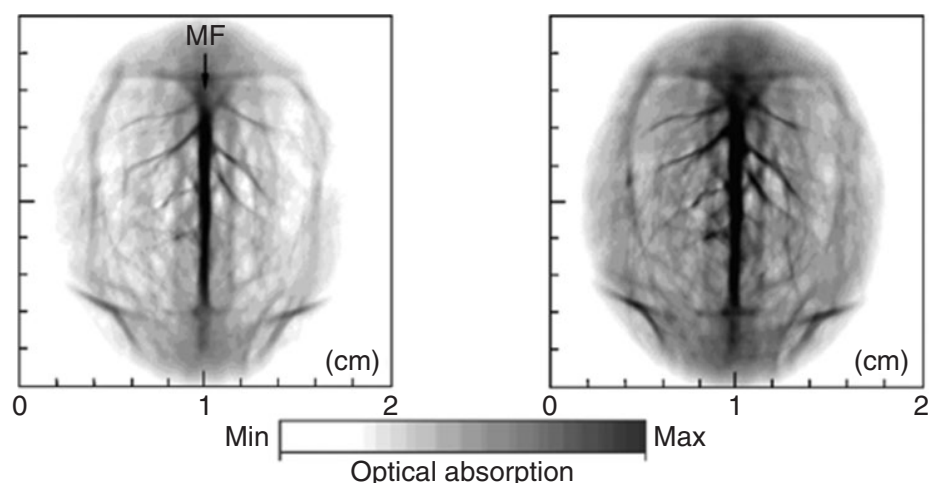

(a)

(b)

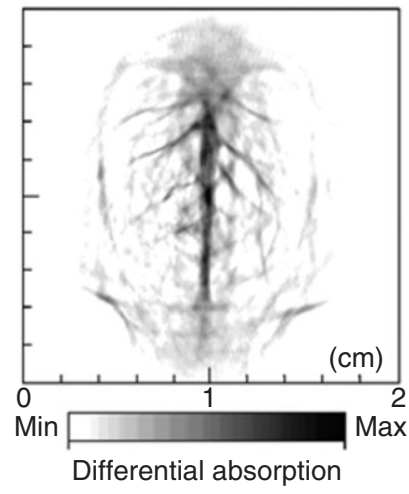

(c)

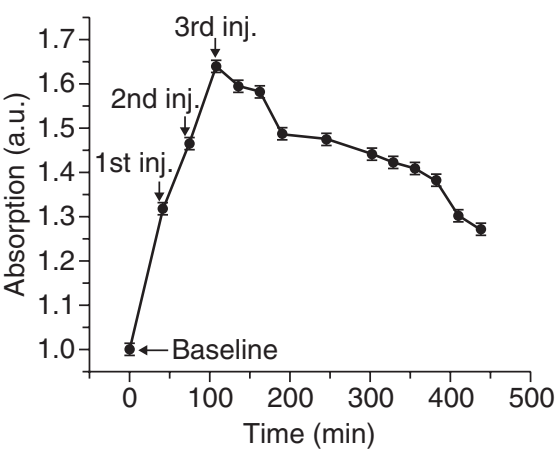

(d) 


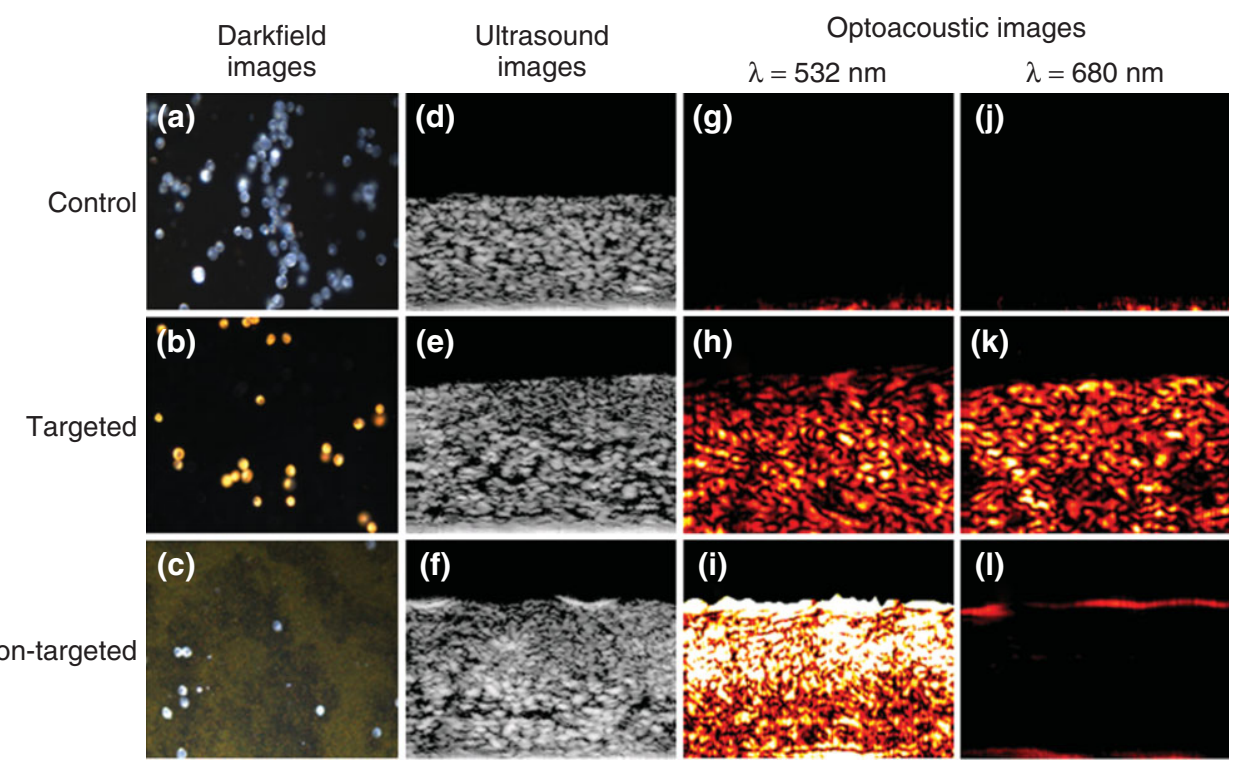

FI G URE 4 | Darkfield, ultrasound and optoacoustic images ( $\lambda=532 \mathrm{~nm}$ and $680 \mathrm{~nm}$ ) of control, targeted and non-targeted tissue phantoms. The darkfield images measure $440 \mu \mathrm{m}$ by $340 \mu \mathrm{m}$ field of view. The ultrasound and optoacoustic images measure $2 \mathrm{~mm}$ by $1.67 \mathrm{~mm}$. (Courtesy of Dr. Emelianov) (Reproduced with permission from Ref 47. Copyright 2007 Optical Society of America).

report showed the feasibility of nanoshells as NIR contrast agents, nanoshells were initially designed for applications in photodynamic therapies. ${ }^{14,23,44}$

Others have shown that nanoshells or other nanoscale particles tend to passively accumulate in tumor regions via the enhanced permeability and retention effect, a mechanism attributed to an increase in vascular porosity. ${ }^{45}$ Indeed, extravasation from healthy vasculature is virtually non-existent, resulting in increased NIR contract in the peripheral tumor vasculature. Reflection-mode $\mathrm{PAI}^{7}$ was used to image nanoshell extravasation from the surrounding vasculature of a subcutaneous murine tumor. ${ }^{46}$ These results showed nanoshell accumulation within the tumor periphery, with minimal accumulation in the tumor core, results which were histologically verified. Furthermore, dynamic studies of nanoshell accumulation in subcutaneous tumors showed eventual clearing from the vasculature, with progressive uptake in the tumor periphery. The contrast ratio between the tumor cortex and the surrounding vasculature was 2 to 1 , indicating that PAI with nanoshell contrast could aid in the detection of cancers. The future direction of this work is focused on integrating PAI and PDT to provide seamless detection and treatment of cancers.

In a study conducted at UT Austin, ${ }^{47}$ PAI was performed at 532 and $680 \mathrm{~nm}$ on three tissue phantoms prepared using A431 skin cancer cells targeted with anti-epidermal growth factor receptor gold bioconjugates (Figure 4). The results of this study demonstrated that using molecular-targeted gold nanoparticles and PAI allowed specific molecular differentiation and highly sensitive and selective detection of skin cancers.
The in vivo detection of gold nanorods (Figure 5) by a PAT system has been recently performed and showed promising results. ${ }^{48}$ The in vivo targeting property of nanorods was also demonstrated for human prostate cancer. ${ }^{49}$ This study described cancer cell targeting by gold nanorods conjugated with an antibody and demonstrated PAI of a single layer of cells. The specific nanoparticle complex was designed for peak absorption in the range of 700-840 nm, optimal for in vivo applications. In a more recent study, gold nanorods were also shown to be capable of targeting multiple molecules. ${ }^{50}$ In this study, two corresponding monoclonal antibodies were conjugated to nanorods with different aspect ratios to target HER2 and CXCR4 molecules. The approach may allow the expression levels of different oncogenes within cancer cells to be revealed simultaneously. Another study used antibody conjugated gold nanorods as a molecular-specific contrast agent to improve the sensitivity of PAI for imaging

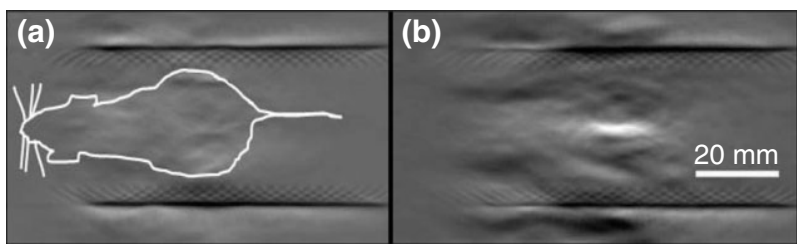

FIG URE 5 | A typical optoacoustic image of a nude mouse before (a) and after (b) subcutaneous injection of $100 \mu \mathrm{L}$ of Au-NRs at a concentration of $7.5 \times 10^{10} \mathrm{NRs}$ per $\mathrm{mL}$ in the abdominal area. Injected nanoparticles were brightly visible in the optoacoustic image

(b).Drawing in (a) depicts the approximate position of the nude mouse during experiment. (Reprinted with permission from Ref 48. Copyright 2007 American Chemical Society). 


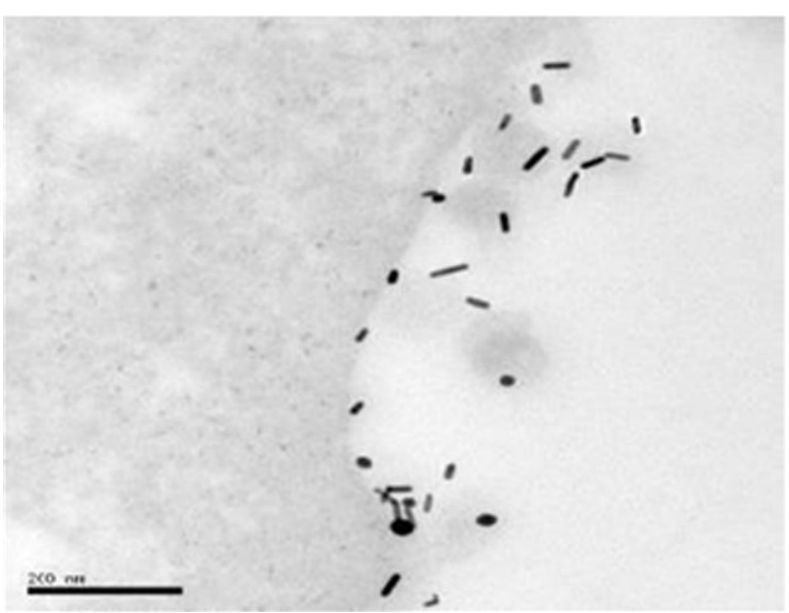

FI G URE 6 | Targeted gold nanoparticles binding to cell membrane. Scale bar: $200 \mathrm{~nm}$ (Courtesy of Dr. Li), (Reproduced with permission from Ref 52. Copyright 2006 SPIE).

of breast tumors. ${ }^{51}$ Cancer cells exhibited enhanced contrast in PAI images because of the presence of nanorods on their surface, whereas the negative control (no nanorods) and cells exposed to nanorods alone (nanorods not conjugated to Herceptin) exhibited no substantial contrast enhancement. Transmission electron microscopy imaging of targeted cells showed effective binding of nanorods to cell membranes (Figure 6) $)^{52}$ and internalization by endocytosis of gold nanospheres (Figure 7). ${ }^{27}$ Antibody conjugated gold nanorods targeted to tumor cells improved the sensitivity of PAI for early detection of deep tumors.

The feasibility of PAI of inflammatory responses using bioconjugated gold nanorods was demonstrated ex vivo. ${ }^{53}$ Gold nanorods were conjugated to antiintercellular adhesion molecule-1, and stimulated endothelial cells labeled with bioconjugated gold nanorods were scanned using a high frequency transducer. The results demonstrated that PAI could differentiate between inflamed cells, which were targeted with PA contrast agents, and control cells.

Owing to the biocompatibility and wellestablished surface chemistry of Au, the optical properties of Au nanocages have also been explored for a number of biomedical applications. ${ }^{33,54}$ For example, it has recently been demonstrated that Au nanocages provide enhanced image contrast when integrated with optical coherence tomography (OCT), an imaging modality capable of resolving tissue microanatomy in vivo using predominantly scattering contrast. ${ }^{55,56}$ Additionally, $\mathrm{Au}$ nanocages functionalized with targeting moieties have been shown to preferentially accumulate on the surface of cancer cells. The subsequent absorption of NIR light by the immuno-targeted

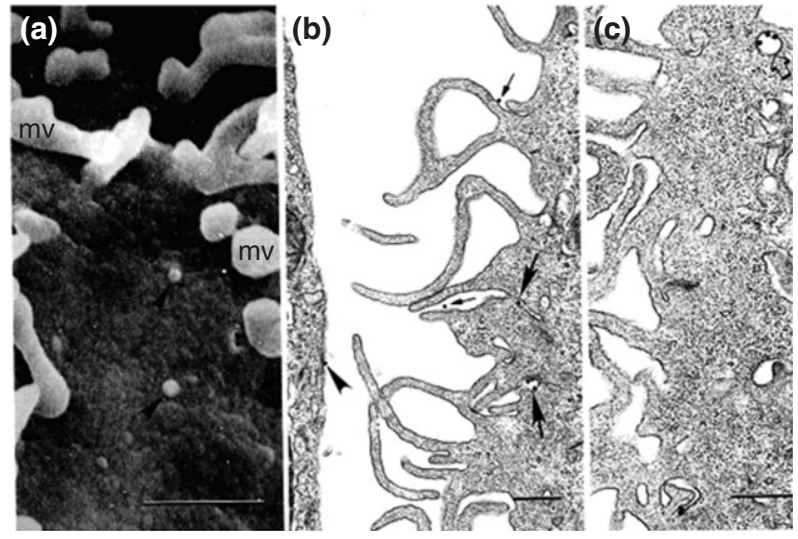

FIG URE 7 | Electron microphotographs of SK-BR-3 cells incubated with Mab/NP. (a) SEM showing two NP (arrowheads) directly on the cell surface. mv - microvilli. (b) TEM of an ultrathin section. NP adherent to the cell surface (arrowhead) and to the surface of microvilli (thin arrows) or in the process of internalization (thick arrows) into vesicles; (c) TEM of an ultrathin section. NP internalized in a cytoplasmic vesicle (open arrow). Scale bars 0.5 micrometers. (Courtesy of Dr. Oraevsky) (Reprinted with permission from Ref 27. Copyright 2004 Elsevier Limited).

$\mathrm{Au}$ nanocages resulted in the selective photothermal destruction of cancer cells in vitro. ${ }^{57}$. A recent in vivo study showed that Au nanocages can effectively enhance PAI when injected into the blood stream during the imaging of a rat cerebral cortex. ${ }^{11}$

ICG-embedded nanoparticles have been conjugated with HER2 antibody for breast cancer and prostate cancer cell targeting. ${ }^{42}$. Initial in vitro characterization shows high contrast and high efficiency for binding to prostate cancer cells. ICG is also a photosensitizer, able to produce highly reactive singlet oxygen by energy transfer from photoexcited dye molecules to oxygen molecules. Photosensitizers were used in PDT as a light-activated cancer therapy. Photosensitization of ICG-ormosil PEBBLEs was found to be suitable for clinical PDT applications.

\section{DISCUSSIONS AND CONCLUSION}

Among the four kinds of nanoparticles employed in PAI so far, nanorods seem to have advantages as optical absorbers. Au nanocages have similar optical absorption to $\mathrm{Au}$ nanorods. Additionally, nanocages are capable of encapsulating drugs. The advantages of ICG-based nanoparticles largely rely on the fact that ICG is the FDA-approved dye for routine clinical use.

All the nanoparticles used are very stable, i.e., non-bleaching, and can last several hours inside the body. There is still a rich array of contrast agents for PAI under testing, such as Au nano colloids, 
carbon nanotubes, etc. All of these contrast agents are non-photobleaching and long-circulating nanoparticles with good optical absorption. Other nanoparticles with strong optical absorption have the potential to become the contrast agents for PAI. However, to date, none of the tested nanoparticles can enhance optical contrast by a factor of two in vivo in the blood stream. A remaining question is whether nanoparticles are safe to use in human beings, although there are no obvious adverse effects reported in small animal experiments. This question will need further study before FDA approval is sought.

As an example of future applications, SPR nanoparticles have started to see use in photothermal therapy. ${ }^{44,58}$ Because SPR nanoparticles are good candidates for PAI, future study in combining PAI and photothermal therapy with the aid of SPR nanoparticles will be very attractive.

The optimal shape of SPR nanoparticles will also need to be studied further. Currently, because of the limited work in PAI, it is impossible to conclude which kind of nanoparticles is best. The chances are that there is probably no 'best'; instead, every kind of nanoparticles may be best suitable for a certain application. In addition, because shape, size, materials, and mechanisms of different nanoparticles vary, proper comparisons between nanoparticles need to be justified.

For future applications, PEBBLEs represent a versatile platform for PAI. Besides enhancing contrast and cell targeting, PEBBLEs can carry sensor dyes and PDT agents. Sensor dyes incorporated in PEBBLEs would allow functional PAI of tissue parameters, such as $\mathrm{pH}$, glucose, dissolved oxygen level, calcium concentration, and cell membrane potential. This would provide clinical in vivo imaging of tissue metabolism and functionality, so far available only for in vitro cellular analysis. PDT dye-embedded PEBBLEs have already been studied for brain tumor targeting and treatment. ${ }^{59}$ Combined with photoacoustic contrast agents, PEBBLEs could provide diagnosis and therapy in a single agent.

In conclusion, advances in nanotechnology have yielded applications in PAI technique. Particularly, the development of nanoscale contrast agents for PAI has allowed researchers to obtain enhanced contrast in the NIR region, where tissue optical absorption is minimized. This capability has afforded applications in deep-tissue imaging. Furthermore, the development of ligand-targeting contrast agents has resulted in the ability to image molecular distributions in vivo with enhanced contrast, potentially improving the ability of early disease detection. Current work in nanoscale contrast agents for PAI is focused on optimizing absorption properties, as well as adding additional functionalities and 'smart' features. This next generation of contrast agents is expected to yield developments in the combination of seamless detection and treatment of diseases.

\section{REFERENCES}

1. Hoelen CGA, de Mul FFM, Pongers R, Dekker A. Three-dimensional photoacoustic imaging of blood vessels in tissue. Opt Lett 1998, 23:648-650.

2. Esenaliev RO, Karabutov AA, Oraevsky AA. Sensitivity of laser opto-acoustic imaging in detection of small deeply embedded tumors. IEEE J Sel Top Quantum Electron 1999, 5:981-988.

3. Kostli KP, Frauchiger D, Niederhauser JJ, Paltauf G, Weber HP, et al. Optoacoustic imaging using a threedimensional reconstruction algorithm. IEEE J Sel Top Quantum Electron 2001, 7:918-923.

4. Kruger RA, Reinecke DR, Kruger GA. Thermoacoustic computed tomography-technical considerations. Med Phys 1999, 26:1832-1837.

5. Wang XD, Pang YJ, Ku G, Xie XY, Stoica G, et al. Noninvasive laser-induced photoacoustic tomography for structural and functional in vivo imaging of the brain. Nat Biotechnol 2003, 21:803-806.
6. $\mathrm{Xu} \mathrm{MH}$, Wang LHV. Photoacoustic imaging in biomedicine. Rev Sci Instrum 2006, 77:041101-1-041101--22.

7. Maslov K, Stoica G, Wang LVH. In vivo dark-field reflection-mode photoacoustic microscopy. Opt Lett 2005, 30:625-627.

8. Zhang HF, Maslov K, Stoica G, Wang LHV. Functional photoacoustic microscopy for high-resolution and noninvasive in vivo imaging. Nat Biotechnol 2006, 24:848-851.

9. Wang YW, Xie XY, Wang XD, Ku G, Gill KL, et al. Photoacoustic tomography of a nanoshell contrast agent in the in vivo rat brain. Nano Lett 2004, 4:1689-1692.

10. Wang XD, Ku G, Wegiel MA, Bornhop DJ, Stoica G, et al. Noninvasive photoacoustic angiography of animal brains in vivo with near-infrared light and an optical contrast agent. Opt Lett 2004, 29:730-732. 
11. Yang X, Skrabalak SE, Li ZY, Xia Y, Wang LV. Photoacoustic tomography of a rat cerebral cortex in vivo with $\mathrm{Au}$ nanocages as an optical contrast agent. Nano Lett 2007, 7:3798-3802.

12. Lin AWH, Lewinski NA, West JL, Halas NJ, Drezek RA. Optically tunable nanoparticle contrast agents for early cancer detection: model-based analysis of gold nanoshells. J Biomed Opt 2005, 10:64035-64010.

13. Jana NR, Gearheart L, Murphy CJ. Seeding growth for size control of 5-40 nm diameter gold nanoparticles. Langmuir 2001, 17:6782-6786.

14. Hirsch L, Gobin A, Lowery A, Tam F, Drezek R, et al. Metal nanoshells. Ann Biomed Eng 2006, 34:15-22.

15. Loo C, Lin A, Hirsch L, Lee MH, Barton J, et al. Nanoshell-enabled photonics-based imaging and therapy of cancer. Technol Cancer Res Treat 2004, $3: 33-40$.

16. Schultz DA. Plasmon resonant particles for biological detection. Curr Opin Biotechnol 2003, 14:13-22.

17. Yang X, Skrabalak S, Stein E, Wu B, Wei X, et al. Photoacoustic tomography with novel optical contrast agents based on gold nanocages or nanoparticles containing near-infrared dyes. Proc SPIE 2008, 6856:68560I.

18. Averitt RD, Sarkar D, Halas NJ. Plasmon resonance shifts of Au-coated $\mathrm{Au}_{2} \mathrm{~S}$ nanoshells: insight into multicomponent nanoparticle growth. Phys Rev Lett 1997, 78:4217.

19. Oldenburg SJ, Averitt RD, Westcott SL, Halas NJ. Nanoengineering of optical resonances. Chem Phys Lett 1998, 288:243-247.

20. Brongersma ML. Nanoshells: gifts in a gold wrapper. Nat Mater 2003, 2:296-297.

21. Oldenburg SJ, Westcott SL, Averitt RD, Halas NJ. Surface enhanced Raman scattering in the near infrared using metal nanoshell substrates. J Chem Phys 1999, 111:4729-4735.

22. Anderson RR, Parrish JA. The optics of human skin. $J$ Invest Dermatol 1981, 77:13-19.

23. Hirsch LR, Stafford RJ, Bankson JA, Sershen SR, Rivera $B$, et al. Nanoshell-mediated near-infrared thermal therapy of tumors under magnetic resonance guidance. Proc Natl Acad Sci U S A 2003, 100:13549-13554.

24. Landsman ML, Kwant G, Mook GA, Zijlstra WG. Light-absorbing properties, stability, and spectral stabilization of indocyanine green. 1976, 575-583.

25. Loo C, Lowery A, Halas N, West J, Drezek R. Immunotargeted nanoshells for integrated cancer imaging and therapy. Nano Lett 2005, 5:709-711.

26. Guzatov DV, Oraevsky AA, Oraevsky AN. Plasmon resonance in ellipsoidal nanoparticles with shells. Quantum Electron 2003, 33:817-822.

27. Copland JA, Eghtedari M, Popov VL, Kotov N, Mamedova $\mathrm{N}$, et al. Bioconjugated gold nanoparticles as a molecular based contrast agent: implications for imaging of deep tumors using optoacoustic tomography. Mol Imaging Biol 2004, 6:341-349.

28. El-Sayed MA. Some interesting properties of metals confined in time and nanometer space of different shapes. Acc Chem Res 2001, 34:257-264.

29. Rao CNR, Kulkarni GU, Thomas PJ, Edwards PP. Metal nanoparticles and their assemblies. Chem Soc Rev 2000, 29:27-35.

30. Sun Y, Xia Y. Shape-controlled synthesis of gold and silver nanoparticles. Science 2002, 298:2176-2179.

31. Chen JY, Wiley B, Li ZY, Campbell D, Saeki F, et al. Gold nanocages: engineering their structure for biomedical applications. Adv Mater 2005, 17:2255-2261.

32. Nehl CL, Hafner JH. Shape-dependent plasmon resonances of gold nanoparticles. 2008, 2415-2419.

33. Hu M, Chen JY, Li ZY, Au L, Hartland GV, et al. Gold nanostructures: engineering their plasmonic properties for biomedical applications. Chem Soc Rev 2006, 35:1084-1094.

34. Martin CR. Membrane-based synthesis of nanomaterials. Chem Mater 1996, 8:1739-1746.

35. Chang SS, Shih CW, Chen CD, Lai WC, Wang CRC. The shape transition of gold nanorods. Langmuir 1999, 15:701-709.

36. Murphy CJ, San TK, Gole AM, Orendorff CJ, Gao JX, et al. Anisotropic metal nanoparticles: synthesis, assembly, and optical applications. J Phys Chem B 2005, 109:13857-13870.

37 Kim F, Song JH, Yang PD. Photochemical synthesis of gold nanorods. J Am Chem Soc 2002, 124:14316-14317.

38. Clark HA, Barker SLR, Brasuel M, Miller MT, Monson E, et al. Subcellular optochemical nanobiosensors: probes encapsulated by biologically localised embedding (PEBBLEs). Sens Actuators B Chem 1998, 51:12-16.

39. Xu H, Yan F, Monson EE, Kopelman R. Roomtemperature preparation and characterization of poly (ethylene glycol)-coated silica nanoparticles for biomedical applications. J Biomed Mater Res A 2003, 66A:870-879.

40. Xu H, Buck SM, Kopelman R, Philbert MA, Brasuel $\mathrm{M}$, et al. Photoexcitation-based nano-explorers: chemical analysis inside live cells and photodynamic therapy. Isr J Chem 2004, 44:317-337.

41. Koo YEL, Cao YF, Kopelman R, Koo SM, Brasuel $\mathrm{M}$, et al. Real-time measurements of dissolved oxygen inside live cells by organically modified silicate fluorescent nanosensors. Anal Chem 2004, 76:2498-2505.

42. Kim G, Huang SW, Day KC, O’Donnell M, Agayan $\mathrm{RR}$, et al. Indocyanine-green-embedded PEBBLEs as a contrast agent for photoacoustic imaging. J Biomed Opt 2007, 12:044020-1-044020-8. 
43. Cao YF, Koo YEL, Koo SM, Kopelman R. Ratiometric singlet oxygen nano-optodes and their use for monitoring photodynamic therapy nanoplatforms. Photochem Photobiol 2005, 81:1489-1498.

44. O’Neal DP, Hirsch LR, Halas NJ, Payne JD, West JL. Photo-thermal tumor ablation in mice using near infrared-absorbing nanoparticles. Cancer Lett 2004, 209:171-176.

45. Maeda H, Fang J, Inutsuka T, Kitamoto Y. Vascular permeability enhancement in solid tumor: various factors, mechanisms involved and its implications. Int Immunopharmacol 2003, 3:319-328.

46. Li M-L, Schwartz JA, Wang J, Stoica G, Wang LV. In-vivo imaging of nanoshell extravasation from solid tumor vasculature by photoacoustic microscopy. In: Oraevsky AA, Wang LV, eds. Photons Plus Ultrasound: Imaging and Sensing 2007: The Eighth Conference on Biomedical Thermoacoustics, Optoacoustics, and Acousto-Optics. Society of Photo-optical Instrumentation Engineers, San Jose, CA; 2007, 64370B.

47. Mallidi S, Larson T, Aaron J, Sokolov K, Emelianov S: Molecular specific optoacoustic imaging with plasmonic nanoparticles. Opt Express 2007, 15:6583.

48. Eghtedari M, Oraevsky A, Copland JA, Kotov NA, Conjusteau A, et al. High sensitivity of in vivo detection of gold nanorods using a laser optoacoustic imaging system. Nano Lett 2007, 7:1914-1918.

49. Agarwal A, Huang SW, O’Donnell M, Day KC, Day M, et al. Targeted gold nanorod contrast agent for prostate cancer detection by photoacoustic imaging. J Appl Phys 2007, 102:064701.

50. Li PC, Wei CW, Liao CK, Chen CD, Pao KC, et al. Photoacoustic imaging of multiple targets using gold nanorods. IEEE Trans Ultrason Ferroelectr Freq Control 2007, 54:1642-1647.
51. Eghtedari M, Copland JA, Kotov NA, Oraevsky AA, Motamedi M. Optoacoustic imaging of nanoparticle labled breast cancer cells: a molecular based approach for imaging of deep tumors. Lasers Surg Med 2004, (Suppl.S16):52-52.

52. Li PC, Wei CW, Liao CK, Chen CD, Pao KC, et al. Multiple targeting in photoacoustic imaging using bioconjugated gold nanorods. Proc SPIE 2006, 6086:60860 M.

53. Kim K, Huang SW, Ashkenazi S, O’Donnell M, Agarwal A, et al. Photoacoustic imaging of early inflammatory response using gold nanorods. Appl Phys Lett 2007, 90:223901-1-223901-3.

54. Skrabalak SE, Chen J, Au L, Lu X, Li X et al. Gold nanocages for biomedical applications. Adv Mater 2007, 19:3177-3184.

55. Cang H, Sun T, Li ZY, Chen JY, Wiley BJ, et al. Gold nanocages as contrast agents for spectroscopic optical coherence tomography. Opt Lett 2005, 30:3048-3050.

56. Chen J, Saeki F, Wiley BJ, Cang H, Cobb MJ, et al. Gold nanocages: bioconjugation and their potential use as optical imaging contrast agents. Nano Lett 2005, 5:473-477.

57. Chen J, Wang D, Xi J, Au L, Siekkinen A, et al. Immuno gold nanocages with tailored optical properties for targeted photothermal destruction of cancer cells. Nano Lett 2007, 7:1318-1322.

58. Zharov VP, Mercer KE, Galitovskaya EN, Smeltzer MS. Photothermal nanotherapeutics and nanodiagnostics for selective killing of bacteria targeted with gold nanoparticles. 2006, 619-627.

59. Reddy GR, Bhojani MS, McConville P, Moody J, Moffat BA, et al. Vascular targeted nanoparticles for imaging and treatment of brain tumors. Clin Cancer Res 2006, 12:6677-6686. 